\title{
A Heuristic Method Based on Answer Set Program in Bilateral Negotiation
}

\author{
GAO Long-yun ${ }^{1, a}$, CHEN Wu ${ }^{1, b, *}$ \\ ${ }^{1}$ College of Computer and Information Science, Southwest University, Chongqing, China \\ a chopin.ring.ly@gmail.com, ${ }^{b}$ chenwu@swu.edu.com \\ *corresponding author
}

\section{Keywords: Answer Set Program, Bilateral Negotiation, Well-Founded Semantics}

\begin{abstract}
This paper presents a heuristic approach based on answer set program in bilateral negotiation. The well-founded semantics for general logic program is used to simplify the process of negotiation. In this approach, demands of each side in negotiation are transformed into a general logic program. The process of negotiation contains two key steps: selecting a pair of answer sets as demand in negotiation and using well-founded semantics to update trade sets of negotiators. Besides, in order to ensure the astringency of approach, some heuristic tips are applied in process. Also, a number of experiments have been done to assess the performance of this method. Results of experiments in general show that this approach could get reasonable results as soon as possible.
\end{abstract}

\section{Introduction}

Negotiation plays a more and more important role in today's social activities. It can take a wide variety of forms, from a trained negotiator acting on behalf of a particular organization, to an informal negotiation between families or friends. Negotiation between two agents is a process of multiple rounds, where some trades are done in each round until a final agreement is reached in general [1]. The last few years have seen a large amount of research which have been done in the field of negotiation. Besides, some researchers are engaged in the optimization in complex negotiations. For example, Colin R.W. at el used Gaussian processes to optimize concession in complex negotiations [2] and Siqi C. at el presented two novel opponent modeling techniques based on deep learning methods [3]. And others also do some research on negotiation method for international business [4]. Furthermore, quite a few research combined with possibilistic logic have been done in the field of multilateral negotiation with incomplete information [5].

In multi-agent system, an in-depth analysis has been done from different perspective in issues of negotiation between agents, such as the mechanism, rules and strategies of negotiation. In the last two decades, another formal method of negotiation has been proposed from the perspective of belief revision. The design of negotiation mechanisms, the design of negotiation rules, the use of negotiation strategy and the flag of successful negotiation are determined by the belief of each intelligent agent [6]. Krister Segerberg proposed irrevocable belief revision, to be contrasted with standard belief revision [7]. A new general framework for default reasoning, which is based on the non-monotonic reasoning framework in the classical first-order language, has been presented by Dongmo $\mathrm{Z}$ at el (2000) [8]. Moreover, there are some different belief revision system under negotiation circumstances for agents raised by other researchers [9].

In recent years, researchers use answer set programming (ASP for short) to present the background knowledge of each agent. A series of studies on mechanisms and methods of both sides in consultation could be conducted by means of logical reasoning. ASP is a form of declarative programming oriented towards difficult (primarily NP-hard) search problems. It is based on the stable model (answer set) semantics of logic programming. In a more general sense, ASP includes all applications of answer sets to knowledge representation [10][11] and the use of Prolog-style query evaluation for solving problems arising in these applications. ASP works out results automatically according to the knowledge base and expected results, and it gives feedback of results to users, which reflects the ability of automated reasoning in artificial intelligence area [12]. An answer set is a 
demand of agents, also a collection of literals essentially. For the feature that ASP has a strong ability in knowledge representation, this paper also presents requirement knowledge of each side in consultations by ASP.

However, these studies does exist imperfections. The biggest flaw in these methods or frameworks is that the balance between optimal results and procedure complexity in negotiation does not been taken into consider. Many researchers find that heuristic method is a useful approach to fix this problem and generate the good enough solutions. At the same time, some studies on heuristic method have been done [13][14].Therefore, profiting from this thought, this paper combines the heuristic method with well-founded semantics based on ASP to optimize the process of negotiation and get the reasonable result. This paper presents a heuristic method in bilateral negotiation. This approach is aimed at the logic-program-based bilateral negotiation and applies some heuristic tips and well-founded semantics to the process of negotiations, which is the main difference compared to others.

\section{Preliminaries}

In this section, each logical program is seen as an agent, and negotiation between the two agents is a procedures of updating demands of each other. Therefore, this chapter introduces the basic concepts of logic answer set and some results, including its semantics, syntax, and other aspects related to the property [12].

Answer set program. The logic program as the most common and the most important way to state knowledge representation plays an indispensable role in knowledge engineering. Most common sense knowledge of human knowledge, its main feature is a non-monotonic reasoning, which happens to be the classic logic insurmountable obstacles. Answer set logic program as an inevitable expansion of classical logic programs, the key idea is to import the default deny "not" to accept "negation as failure" semantics. This section mainly describes the logic program which is related to negotiation closely: Extended Logic Program (ELP).

Traditional logic program has a great weakness is that you are not allowed to process directly with incomplete information. The requests in traditional logic program miss corresponding results to undecidable words, and these words express the incompleteness of the information non classical logic theory. To overcome these shortcomings, the literature [15] proposed extended logic programs, in addition to "negation as failure (not)", it also introduced the "classical negation (neg)". Therefore, ELP not only gets hidden negative information through the "closed world assumption", but also includes clear negative information. The following context describes the simple grammar of ELP.

Let $\mathcal{L}$ represent ELP's language. The Herband domain of language $\mathcal{L}$ is a collection of all the basic atoms. And basic atoms are composed of the constants and functions appeared in the language L. This paper uses $U_{\mathcal{L}}$ to express them. Basic atoms of Herband domain in language $\mathcal{L}$ are formed by predicate symbols. Predicated symbols are appeared in $\mathcal{L}$ and set of all the basic atoms are generated by the atoms of Herband domain, which is expressed by $U_{\mathcal{L}}$. A normal rule usually has the form of the following.

$$
L_{0} \leftarrow L_{1}, \ldots, L_{m}, \text { not } L_{m+1}, \ldots, \text { not } L_{n}(n \geq m \geq 1)
$$

We use $C_{n}(P)$ to represent the answer set of basic program $P$. To calculate the ELP's answer set, guessing a set of literals in the ELP should been done firstly. Then this method should analysis if this program could be converted into a Classical Logic Program (GL Statute) or not. Next, we should calculate the answer set of the basic logical program. At last, this approach must detect the consistency of generated answer set with the original literals set. For more information about the answer set program, readers can refer to the literature [12].

Belief Revision. Multi-round negotiation is a process of mutual modification of two ELPs' negotiation needs (answer sets). The method divide the process into two parts: contraction and expansion. Contraction of answer set refers to the answer set to give up their own part of the literals. Expansion of answer set means that the one negotiator accepts some literals of another negotiator's 
answer set. Expansion is achieved by taking some literals from another negotiator's answer set as new facts into its own program.

The literals which negotiators choose to give up are represented as $G_{i}$. The literals which negotiators choose to retain are represented as $\mathrm{R} \_\mathrm{i}$. For an ELP $\Pi$, this paper defines $\left(R_{i}, G_{i}\right)$ as its preference. For $E L P \Pi_{1}$ and $E L P \Pi_{2}$, this paper uses $\left(R_{1}, R_{2}\right)$ to express the trade set of negotiation.

Well-Founded Semantics. The well-founded semantics of a program $P$ is the "meaning" represented by the least fixed point of $\mathrm{W}_{\mathrm{P}}$. Every positive literal denotes that its atom is true, every negative literal denotes that its atom is false, and missing atoms have no truth value assigned by the semantics [17].

Researchers generally believe that well-founded semantics are reasonable three-valued semantics, with a series of excellent characteristics: Each logic program has a unique well-founded semantics; well-founded semantics and a series of special types of logic programs recognized regular semantic equivalents such as, well-founded hierarchical logic program semantics equivalent to their perfect semantics [17], and perfect semantics are considered hierarchical logic regular semantics [12]; well-founded semantics includes support semantic characteristics [18] the so-called support semantic It refers to any of the semantics of a basic text are from the extended logic programs in a corresponding rule reasoning of this feature to ensure the effectiveness of the security policy describes security requirements; existence of efficient semantic semantics based on well-founded search algorithm with good usability, such as SLG algorithm [19][20]. So this paper chooses well-founded semantics to simply the process of updating trade sets in bilateral negotiation.

\section{Heuristic Method in Bilateral Negotiation}

We mainly describe basic concepts and the process of answer set solving in previous sections. This paper proposes a heuristic method in bilateral negotiation. And this method mainly consists of two key steps, selecting a pair of answer sets as demand in negotiation and updating trade sets of negotiators.

Selecting Initial Demand. This step contains following five items.

(1) Transform the each agent's demands described by natural language into $E L P, \Pi_{1}$ and $\Pi_{2}$

(2) Use GL Statute to convert ELP into Classical Logic Programs.

(3) Calculate answer sets of each Classical Logic Program. If there is no answer sets for any negotiator, the negotiation failed. Or, take the next step.

(4) Sort the answer sets of each negotiators in descending order. The rules of sorting is as below.

(a) An rational agent should accept as much demands as possible. So comparing the number of literals in one answer set to another answer set has to be done. The bigger the number, the better the answer set.

(b) If the number of one answer set is equal with the number of another answer set, the approach calculates the sum of importance of all the extended literals(excluding the facts in answer sets) by following formula:

$D(v)=\sum\left(n_{i j}(v) / n_{i j}\right)(i \neq j)$

(2)

Where $D(v)$ represents the important values (betweenness) of node (literal), $n_{i j}$ is the number of shortest paths between node $\mathrm{i}$ and node $\mathrm{j}$.

The results of this step is as following.

$$
\left\{S_{0}, S_{1}, \ldots, S_{m}\right\},\left\{S_{0}^{\prime}, S_{1}^{\prime}, \ldots, S_{k}^{\prime}\right\}, m \geq 0, k \geq 0
$$

(3)

(5) Choose the initial answer sets of two negotiators, $\left(S_{0}, S_{0}^{\prime}\right)$.

(6) Calculate the well-founded sets, unfounded sets and the rest sets of each negotiators by the well-founded semantics. This paper uses WF to present well-founded sets, UF to present unfounded sets and $R T$ represent the rest sets.This step generates the following results, $W F, W F^{\prime}, U F, U F^{\prime}, R T, R T^{\prime}$.

In this part, graph theory is used to express rules in original demands. Agent choose demands with larger betweenness to negotiate and give up demand with smaller betweenness in order to make a deal. Owning the bigger sum means the answer set has a higher priority. 
With the sorting is finished, the method chooses first answer sets from each descending order separately. And these two answer sets are regarded as the initial answer sets of negotiation. After all the above steps, the initial answer sets of two negotiators, $\left(S_{0}, S_{0}{ }^{\prime}\right)$, can be get. Then method continues to take next step of updating trade sets.

Updating Trade Sets. For each negotiator, trade set consists of two parts. One is a set of accepted literals. Another is a set of abandoned literals. By the first key step, the well-founded sets, unfounded sets and the rest sets of each negotiator are generated. In this paper, WF is regard as core demands of negotiator that must be retained; UF is regard as useless demands that must be discarded; RT is regard as negotiable demands that can be either retained or discarded. Besides, the accepted set should include literals as important as possible. To achieve this goal, ranking literals by their importance should be done. The method of ranking is shown as formula 2. As for abandoned literals, they must be inconsistent literals of two initial answer sets. Maybe one of negotiators choose to give up the inconsistent literal or literals.

By the process of step one, the initial answer sets $\left(S_{0}, S_{0}{ }^{\prime}\right)$ as usual could be get. If negotiators cannot reach an agreement with initial answer sets, the method should update trade set. The process is as follows.

(1) If there is no agreement reached in condition that one of negotiators gives up inconsistent literals, both of negotiators drop inconsistent literals.

(2) Update the accepted literals by their rankings. If the most important literal can not generate accepted result, less important literal is used to replace it. The rest can be deduced by analogy.

(3) If above processes do not work, negotiation failed.

\section{Experiments and Results}

This section uses an example to explain how this approach works. Here is the description of negotiation between trade union and capital, which is in natural language.

Example 1.Trade union and employer would like to negotiate the new labor contract. Trade union is inclined to increase the workers' salary and welfare. But employer intends to quit some workers, and to adopt new production technology. The following is the basis of the negotiation.

If employer quits workers, trade union will strikes; but trade union thinks that employer don't want to strike. If employer don't want to strike, they will agree to increase the workers' salary and welfare. In order to increase the profit, employer would like to use new technology and quit some workers. Because increasing investment and higher wages will lead to cost increased. Trade union knows that if employer to adopt new technology, some workers will be unemployed. So trade union hopes that employer increases the investment to provide enough jobs, so that workers do not be fired. If employer quits workers, trade union will strike. Employer knows that increasing investment will bring more profits in the future. On the other hand, employer knows that increasing the current profit only if workers don't strike and don't increase wages.

We transforms natural description into ELP, which is shown as Table 1.

Table 1. ELP of trade union and capital

\begin{tabular}{|c|c|c|}
\hline Negotiator & trade union $\left(\boldsymbol{\Pi}_{\mathbf{1}}\right)$ & $\operatorname{capital}\left(\boldsymbol{\Pi}_{2}\right)$ \\
\hline ELPS & $\begin{array}{l}\text { strike } \leftarrow \text { not } r a i s e \_s a l, n o t \text { raise_wel. } \\
\text { raise_sal } \leftarrow \text { not strike. } \\
\text { raise_wel } \leftarrow \text { not strike. } \\
\text { inc_invest } \leftarrow \text { not strike. } \\
\text { strike } \leftarrow \text { lay_off. } \\
\text { lay_off } \leftarrow \text { inc_invest. }\end{array}$ & $\begin{array}{l}\text { new_tech } \leftarrow \text { not strike. } \\
\text { lay_off } \leftarrow \text { new_tech,not inc_invest. } \\
\text { red_cost } \leftarrow \text { lay_off. } \\
\neg r e d \_c o s t \leftarrow r a i s e \_s a l . \\
r e d \_c o s t \leftarrow \text { lay_off. } \\
\text { c_profit } \leftarrow \text { red_cost,not_strike,not raise_sal. }\end{array}$ \\
\hline
\end{tabular}

In this approach, following agreement of negotiation can be generated with a little time cost. When negotiators choose ( $\left\{\right.$ raise_sal,raise_wel,inc_invest, $\left.\left.\neg l a y_{-} o f f\right\},\left\{n e w_{-} t e c h\right\}\right)$ as their trade set, the example can get the following agreement, raise_wel, $\neg l a y \_o f f, n e w_{-} t e c h, r e d \_c o s t, c_{-}$profit. Through this method, negotiators can save much more times than using traditional approaches in general. In worst case, it spends as same time as traditional approaches. The experiments show that the heuristic method has an outstanding performance in general. 


\section{Summary}

This paper proposes a heuristic method of bilateral negotiation based on answer set programming. And the astringency of this method is ensured by selecting only one pair of initial answer set to negotiate in first step and accepting literals as important as possible. This paper also defines its own ranking criteria in answer set sorting. It's clear that this method could get a reasonable result of negotiations by accepting literals which is more important to negotiators. Moreover, this method could take less time to finish the negotiation than traditional approaches. Especially, this method could be used in the negotiation of buyers and sellers in e-commerce.

Currently this paper only consider the primary bilateral negotiations, and do not take the time constraint and other constraints of negotiation and multilateral negotiations into consideration. As for future work, extending this method to the multilateral negotiations and adding time constraint are under way. Besides, taking the external influence into consideration [16] is in the plan. In addition, the optimization of benefits criteria still has more work to be done.

\section{References}

[1] Hussain I, Knapen L, Galland S, et al. Organizational and agent-based automated negotiation model for carpooling. Procedia Computer Science Vol.37(2014), p. 396-403.

[2] Williams C R, Robu V, Gerding E $\mathrm{H}$, et al. Using gaussian processes to optimise concession in complex negotiations against unknown opponents(2011).

[3] Chen S, Bou-Ammar H, Tuyls K, et al. Conditional Restricted Boltzmann Machines for Negotiations in Highly Competitive and Complex Domains(IJCAI 2013).

[4] Peleckis K. International business negotiations: innovation, negotiation team, preparation. Procedia-Social and Behavioral Sciences Vol.110(2014), p. 64-73.

[5] De Clercq S, Schockaert S, Nowé A, et al. Multilateral negotiation in Boolean games with incomplete information using generalized possibilistic logic. Proceedings of the 24th International Conference on Artificial Intelligence. AAAI Press (2015), p. 2890-2896.

[6] Chen W, Zhang M Y, Wu M N. A Logic-Program-Based Negotiation Mechanism. Journal of Computer Science and Technology (2009) Vol.24(4), p. 753-760.

[7] Segerberg K. Irrevocable belief revision in dynamic doxastic logic. Notre Dame journal of formal logic (1998) Vol.39(3), p. 287-306.

[8] Zhang D, Zhu Z, Chen S. Default reasoning and belief revision: a syntax-independent approach. Journal of Computer Science and Technology (2000) Vol.15(5), p. 430-438.

[9] Enqvist S. Interrogative belief revision in modal logic. Journal of philosophical logic (2009) Vol.38(5), p. 527-548.

[10] Baral C. Knowledge representation, reasoning and declarative problem solving. Cambridge university press(2003).

[11] Van Harmelen Frank, Lifschitz Vladimir, Porter Bruce. Handbook of knowledge representation. Elsevier Vol.1(2008), p. 285-316.

[12] Lifschitz V. Foundations of logic programming. Principles of knowledge representation Vol.3(1996), p. 69-127.

[13] Costantini S, De Gasperis G, Provetti A, et al. A heuristic approach to proposal-based negotiation: with applications in fashion supply chain management. Mathematical Problems in Engineering(2013).

[14] Costantini S, Tocchio A, Tsintza P. A Heuristic Approach to P2P Negotiation. Computational Logic in Multi-Agent Systems. Springer Berlin Heidelberg (2007), p. 177-192.

[15] Gelfond M, Lifschitz V. Classical negation in logic programs and disjunctive databases. New generation computing (1991) Vol.9(3-4), p. 365-385.

[16] Lin R, Kraus S, Wilkenfeld J, et al. Negotiating with bounded rational agents in environments with incomplete information using an automated agent. Artificial Intelligence(2008) Vol.172(6), p. 823-851.

[17] Van Gelder, Allen, Kenneth A. Ross, and John S. Schlipf. The well-founded semantics for 
general logic programs. Journal of the ACM (JACM 1991) Vol.38(3): p. 619-649.

[18] Przymusinski T. Well-Founded semantics coincides with three-valued stable semantics. Fundamenta Informaticae(1990) Vol.13(4), p. 445-463.

[19] Chen W, Warren D S. Query evaluation under the well-founded semantics. Proceedings of the twelfth ACM SIGACT-SIGMOD-SIGART symposium on Principles of database systems. ACM (1993), p. 168-179.

[20] Bell D E, La Padula L J. Secure computer system: Unified exposition and multics interpretation. (MITRE CORP BEDFORD MA 1976). 